\title{
REDUCTIVE ALGEBRAS AND AUTOMORPHISM GROUPS OF VON NEUMANN ALGEBRAS
}

\author{
BY RICHARD I. LOEBL AND PAUL S. MUHLY ${ }^{1}$ \\ Communicated by Paul Halmos, March 3, 1975
}

An algebra $\mathfrak{U}$ of operators on a Hilbert space $H$ is called reductive in case every subspace of $H$ invariant under $\mathbb{U}$ is invariant under $\mathfrak{U}^{*}$. Our objective in this note is to indicate how to construct some nonselfadjoint, ultraweakly closed, reductive algebras. Our algebras do not provide a counterexample to the reductive algebra question: Are there any nonselfadjoint, weakly closed, reductive algebras? because each is weakly dense in the von Neumann algebra it generates. However, they do provide evidence for a negative answer to the question.

Let $\mathfrak{B}$ be a von Neumann algebra on the Hilbert space $H$, let $\left\{\alpha_{t}\right\}_{t \in R}$ be an ultraweakly continuous, one-parameter group of $*$-automorphisms of $\mathfrak{B}$, and let $\mathfrak{U}$ be the set of $A \in \mathfrak{B}$ such that for each ultraweakly continuous linear functional $\rho$ on $\mathfrak{B}$ the function of $t, \rho\left(\alpha_{t}(A)\right)$, admits a bounded analytic extension to the upper half-plane. Then $\mathfrak{U}$ is an ultraweakly closed subalgebra of $\mathfrak{B}$ which generates $\mathfrak{B}$ such that $\mathfrak{U} \cap \mathfrak{U}^{*}=\left\{A \in \mathfrak{B} \mid \alpha_{t}(A)=A\right.$, for all $\left.t \in \mathbf{R}\right\}$ [1].

The following theorem goes back to Helson and Lowdenslager [3] and its proof may be constructed easily from Arveson's paper [1] or from Forelli's [2].

THEOREM 1. Let $M$ be a closed subspace of $H$ invariant under $\mathfrak{U}$, but not under $\mathfrak{B}$. Then there is a nonzero projection $e$ in $\mathfrak{B}^{\prime}$, and there is a strongly continuous unitary representation $\left\{U_{t}\right\}_{t \in \mathbf{R}}$ of $\mathbf{R}$ on eH such that

$$
U_{t}(e A e) U_{t}^{*}=e \alpha_{t}(A) e, \quad A \in \mathfrak{B}, \quad t \in \mathbf{R} .
$$

Moreover, $E([0, \infty)) e H \supseteq e M \supseteq E((0, \infty)) H$, where $E$ is the spectral measure of $\left\{U_{t}\right\}_{t \in \mathbf{R}}$.

AMS(MOS) subject classifications (1970). Primary 46L15, 46L10; Secondary 22D10, 43A65.

1 Partially supported by a grant from the National Science Foundation. 
There is a converse, but it will not be needed for our examples.

It follows that $\mathfrak{U}$ will be reductive whenever it is impossible to find a subspace invariant under $\mathfrak{B}$ on which $\left\{\alpha_{t}\right\}_{t \in \mathbf{R}}$ is unitarily implemented; i.e., on which there is a unitary representation of $\mathbf{R}$ satisfying (*). The following theorem provides criteria for deciding when this happens. It was shown to us by Masamichi Takesaki who based the proof on an argument of Kadison [4].

THEOREM 2. Let $\mathfrak{B}$ be a $\mathrm{II}_{\infty}$ factor with faithful normal trace tr. Suppose $\mathfrak{B}^{\prime}$ is finite and that there is a real $\lambda(\neq 0)$ such that $\operatorname{tr} \circ \alpha_{t}=e^{\lambda t} \operatorname{tr}$ for all $t \in \mathbf{R}$. Then $\left\{\alpha_{t}\right\}_{t \in \mathbf{R}}$ cannot be unitariiy implemented on any subspace invariant under $\mathfrak{B}$.

Surprisingly, examples of factors and automorphism groups satisfying the hypotheses of Theorem 2 abound in nature (cf. [6]); however, they are often difficult to exhibit explicitly. A reasonably tractable concrete example, shown to us by Takesaki, can be constructed with a variant of the group-measure construction of Murray and von Neumann.

As we mentioned above, our examples are weakly dense in their containing von Neumann algebras. To see this, observe that if $\mathfrak{B}$ and $\left\{\alpha_{t}\right\}_{t \in R}$ satisfy the hypotheses of Theorem 2 , then any finite ampliation of $\mathfrak{B}$ together with the automorphism group induced by $\left\{\alpha_{t}\right\}_{t \in \mathbf{R}}$ also satisfies the hypotheses of Theorem 2. From this, it follows that every finite ampliation of $\mathfrak{U}$ has the same invariant subspaces as the corresponding ampliation of $\mathfrak{B}$; whence $\mathfrak{U}$ is weakly dense in $\mathfrak{B}$. No matter what the final answer to the reductive algebra question is, our examples place in sharp relief once again differences between the weak and ultraweak topologies-differences not anticipated by conventional wisdom.

ACKNOwLEDgement. It is with great pleasure that we thank Masamichi Takesaki for correspondence which was valuable to us in the preparation of this note.

\section{REFERENCES}

1. W. B. Arveson, On groups of automorphisms of operator algebras, J. Functional Analysis 15 (1974), 217-243.

2. F. Forelli, Analytic and quasi-invariant measures, Acta Math. 118 (1967), 3359. MR 35 \#667.

3. H. Helson and D. Lowdenslager, Invariant subspaces, Proc. Internat. Sympos. Linear Spaces (Jerusalem, 1960), Jerusalem Academic Press, Jerusalem; Pergamon, Oxford, 1961, pp. 251-262. MR 28 \#487. 
4. R. V. Kadison, Isomorphisms of factors of infinite type, Canad. J. Math. 7 (1955), 322-327. MR 17, 178.

5. R. I. Loebl and P. S. Muhly, Analyticity and flows on von Neumann algebras (in preparation).

6. M. Takesaki, Duality for crossed products and the structure of von Neumann algebras of type III, Acta Math. 131 (1973), 249-310.

DEPARTMENT OF MATHEMATICS, WAYNE STATE UNIVERSITY, DETROIT, MICHIGAN 48202

DIVISION OF MATHEMATICAL SCIENCES, UNIVERSITY OF IOWA, IOWA CITY, IOWA 52242

BULLETIN OF THE

AMERICAN MATHEMATICAL SOCIETY

Volume 81 , Number 4 , July 1975

\section{INTEGRAL TRANSFORMS OF WEAK TYPE BETWEEN REARRANGEMENT INVARIANT SPACES}

BY MARIO MILMAN

Communicated March 20, 1975

1. Introduction. Let $X(\Omega), Y(\Omega)$ and $Z(\Omega \times \Omega)$ be rearrangement invariant Banach function spaces, where $\Omega=(0, \infty)$ with the Lebesgue measure. Let $M(\Omega)$ be the set of measurable functions on $\Omega$ and for every $k \in Z(\Omega \times \Omega)$, denote by $z_{k}$ the integral operator given by $z_{k}(f)(x)=\int_{\Omega} k(x, y) f(y) d y$ for $f \in M(\Omega), x \in \Omega$.

In this paper we shall give necessary and sufficient conditions, in terms of the fundamental functions of the spaces (see [2] and [4]) for $z_{k}$ to be of weak type $\{X, Y\}$ for every $k \in Z$. The methods are similar to those employed by $O$ 'Neil in his fundamental paper [3].

2. The Lorentz $\Lambda(Z)$ and $M(Z)$ spaces. It is well known how to define the Lorentz $\Lambda$ and $M$ spaces associated with $X(\Omega)$. To extend these definitions to $Z(\Omega \times \Omega)$, we "smash" $Z$ into $\hat{Z}(\Omega)$, say, via Luxemburg's representation theorem [1]. The relationship between the fundamental functions of these 